FORMATION Formation emploi

Revue française de sciences sociales

143 | Juillet-Septembre 2018

Le retour en formation : une vraie chance?

\title{
Présentation de l'ouvrage de S. Beaud, La France des Belhoumi. Portraits de famille (1977-2017)
}

Henri Eckert

\section{(2) OpenEdition \\ 12 Journals}

Édition électronique

URL : http://journals.openedition.org/formationemploi/6378

DOI : 10.4000/formationemploi.6378

ISSN : 2107-0946

Éditeur

La Documentation française

Édition imprimée

Date de publication : 20 novembre 2018

Pagination : 213-219

ISSN : 0759-6340

Référence électronique

Henri Eckert, «Présentation de l'ouvrage de S. Beaud, La France des Belhoumi. Portraits de famille (1977-2017) », Formation emploi [En ligne], 143 | Juillet-Septembre 2018, mis en ligne le 20 novembre 2018, consulté le 07 novembre 2020. URL : http://journals.openedition.org/formationemploi/6378 ; DOI : https://doi.org/10.4000/formationemploi.6378 


\title{
Note de lecture
}

\section{Présentation de l'ouvrage de S. Beaud, La France des Belhoumi. Portraits de famille (1977-2017)}

\author{
Henri Eckert \\ Professeur émérite de sociologie à l'Université de Poitiers
}

Samira, Leila, Amel et les autres: histoire de l'intégration d'une famille immigrée dans la France du tournant du siècle

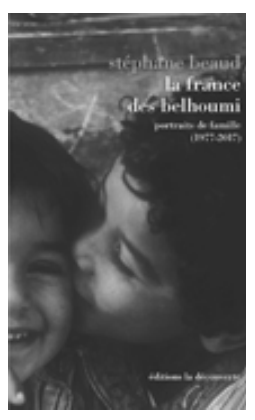

Nous sommes en juin 2012, dans une commune de Seine-Saint-Denis; la Mission locale pour l'emploi fête son trentième anniversaire. Invité à parler de l'insertion professionnelle des jeunes de milieu populaire, Stéphane Beaud, au moment de quitter la salle à la fin de la réunion, est retenu par trois jeunes femmes, visiblement désireuses de poursuivre avec lui la discussion entamée plus tôt. L'ainée, puisqu'il s’agit de trois sœurs, le remercie pour son exposé et, plus précisément, d'avoir insisté, au cours de la discussion, sur "la différenciation interne du groupe des descendants d'immigrés maghrébins" (p. 8), dont les trois font partie.

Il faut, pour saisir toute l'importance de cette appréciation, prendre en compte le contexte socio-politique de l'époque : l'actualité récente avait été marquée, sur le fond des émeutes qui avaient agité les banlieues des grandes villes françaises en 2005, par les attaques menées par Mohamed Merah contre une école juive et une caserne à Toulouse, qui avaient provoqué sept morts, et les discours vindicatifs sinon agressifs sur "l'identité nationale ", tenus par le candidat de la droite à l'élection présidentielle de 2012.

Ainsi, les musulmans s'avéreraient incapables de s'intégrer dans la société française, notamment les enfants de ceux que la France avait appelés dans ses usines au temps de sa forte croissance économique. Ceux, donc, que rassemblaient des expressions comme "enfants d'immigrés » ou " deuxième génération ", plus récemment : « jeunes des banlieues » ou «jeunes des cités».

C'est contre ce point de vue que se dresse le livre, contre les représentations construites par des instances qui ne cessent de monter en épingle des événements parfois réels, mais souvent fantasmés ou déformés pour, prétendent-elles ou insinuent-elles subrepticement, démontrer que ces jeunes-là seraient décidément incapables de s’intégrer. 
Le livre de Stéphane Beaud propose ainsi une contre-image, "beaucoup plus réaliste sociologiquement que l'image, spectaculaire et médiatique, qui s'est diffusée et enkystée dans maints segments de la société française, celle d'un groupe social réputé "inassimilable " (p. 11) ${ }^{1}$. Il s'attache, dans ce but, à restituer l'histoire d'une famille immigrée, en France, au début des années 1970, dont les enfants ont atteint progressivement des positions sociales confortables, pour le moins stabilisées, et se comportent globalement comme leurs concitoyens.

Cette histoire a été reconstituée patiemment, au fil d'une longue enquête par entretiens, menée entre 2012 et 2017, avec la complicité active des sœurs ainées de la fratrie, qui ont, en quelque sorte, joué le rôle d' "informatrices privilégiées " sinon d' " alliées " du sociologue. Stéphane Beaud parle ainsi d'un "pacte " noué avec les trois sœurs : "Il s'agissait de raconter - et de leur faire raconter - l'histoire ordinaire d'une famille algérienne ordinaire en France" (p. 12). Il va jusqu'à écrire, un peu plus loin, que c’est Samira, la sœur ainée de la fratrie, qui aurait «voulu» (p. 12) l'enquête !

Menée sur une durée de cinq années elle a, sans surprise, été affectée tant par sa dynamique propre, prenant progressivement « un tour plus collaboratif» (p. 13), que par les événements qui se sont produits entre temps, les terribles attentats perpétrés à Paris ou à Nice en 2015, pour « suivre dans le temps et de plus près la manière dont les membres de la fratrie vivaient ces événements, les subissaient etlou y réagissaient, en les invitant aussi à réfléchir avec (le sociologue) sur le rapport qu'ils entretenaient à leur avenir et leur condition sociale " (p. 13).

Dans la mesure où le livre se présente comme une étude de cas, l'étude d'une famille algérienne immigrée en France, se pose nécessairement la question de la possibilité de généraliser, sur la base des observations faites, les analyses qui en ont été tirées. Stéphane Beaud aborde la question dans la conclusion de l'ouvrage et nous y reviendrons à la fin de cette note. Dans l'immédiat, nous nous intéresserons aux grandes étapes de la vie sociale des protagonistes de cette histoire familiale et plus particulièrement aux scolarités des enfants Belhoumi, à leurs insertions professionnelles et leurs devenirs sociaux.

\section{Une famille venue d'Algérie s'installer en France}

Le père, qui avait opté pour la nationalité algérienne après l'indépendance de son pays, obtient le " ticket ", indispensable pour aller travailler en France, rapidement après son mariage ; il espère y trouver des conditions d'emploi, de travail et de vie plus satisfaisantes (chap. 1). Son épouse le rejoint quelques années plus tard, en 1977, dans le cadre du regroupement familial ; elle emporte avec elle leurs trois premiers enfants nés en Algérie, deux filles suivies d'un garçon. L'aînée des trois avait entamé sa scolarité primaire en Algérie et arrive en France sans parler un mot de français ; elle comblera très vite son

1 Voir aussi l'article de Stéphane Beaud paru dans Le monde diplomatique, en avril 2018, sous le titre Une famille algérienne de France. 
handicap et poursuivra une scolarité exemplaire. Puis naîtront successivement deux autres garçons et trois filles.

La fratrie s'organise ainsi en trois groupes, au croisement du sexe et de l'âge : les deux filles ainées, puis les trois garçons et, enfin, les trois cadettes. Seize ans séparent la plus âgée de la plus jeune des cinq filles. De cet écart résulte que les enfants n’ont pas été scolarisés tout à fait dans les mêmes conditions et, à cette différence, se superpose celles liées à la divergence des comportements scolaires des garçons et des filles. Si les filles ont, globalement, suivi des scolarités pour le moins honorables, il n'en va pas de même pour les trois garçons : l'un d'entre eux seulement obtient un baccalauréat, professionnel, alors que les filles ont toutes obtenu un baccalauréat général ou technologique avant de poursuivre des études supérieures. Stéphane Beaud avance, entre autres explications, les socialisations différentes des garçons et des filles : alors que celles-ci, pour échapper à l'enfermement domestique subi et/ou promis aux femmes, s'ouvriraient leur horizon de vie par la réussite scolaire notamment, les garçons ne tireraient guère de bénéfice des libertés et des privilèges dont ils disposent au sein de la famille et s'en trouveraient piégés par l'échec scolaire. Les modalités plus spécifiques de socialisation rendent toutefois compte d'autres différences, par exemple entre les filles elles-mêmes.

S’impose dès lors la nécessité d'examiner plus attentivement les trajectoires des enfants de la famille Belhoumi, d'autant qu'une riche monographie en permet une description très fine. Nous prendrons ici l'exemple des sœurs aînées (chap. 2).

Si Samira apprend rapidement la langue française et devient une très bonne élève dès l'école primaire, ce n'est pas seulement parce qu'elle est une fille : elle goûte très tôt au plaisir de la langue française et, surtout, fréquente les bibliothèques de l'école ou du quartier. Elle " dévore les livres». Elle bénéficie aussi du soutien chaleureux de ses enseignants, de l'une d'entre elles en particulier, avec qui elle a maintenu le contact depuis. Malgré les corvées domestiques, que la tradition familiale assigne aux filles, et une vie coincée entre l'école et la maison, elle prend très tôt conscience, dit-elle, du fait "que le passeport pour (son) émancipation, c'était la réussite scolaire» (p. 57). Elle est, en cela, soutenu par un père qui, arrivé en France, a dû "travailler avec la pioche ", selon ses propres mots, mais rêve pour ses enfants qu'ils puissent "travailler avec le stylo". Ainsi Samira ouvre la voie à ses frères et sœurs, elle " trace le sillon ».

Leïla, sa cadette immédiate, a certes suivi un chemin quelque peu différent ; il ne lui assurera pas moins la réussite scolaire, mais aussi l'ouverture de perspectives sociales et professionnelles. Acceptant moins volontiers que sa sœur d'être recluse dans l'espace familial, elle déploie au contraire des stratégies "pour échapper à l'enfermement domestique " (p. 64) en s'engageant dans des activités sportives et l'animation socioculturelle. Elle tire parti de l'offre de loisirs d'une commune alors gérée par le Parti communiste français et c'est « moins l'école et sa trajectoire scolaire que l'environnement sociopolitique de la ville dans laquelle elle a grandi qui l'a fait, à ses yeux, devenir ce qu'elle est" (p. 65). C'est ainsi qu'elle s'engage un peu plus tard dans l'activité professionnelle de chargée de projets d'insertion 
en mission locale, tirant parti de son expérience dans le milieu associatif de son enfance, alors que son aînée, après avoir intégré une école d'infirmière, a, depuis, accédé à la position de cadre de santé.

\section{La réussite scolaire comme vecteur de mobilité sociale ascendante}

Si l'analyse plus serrée des parcours de Samira et Leila met en évidence leur désir d'acculturation, malgré les difficultés qu'elles ont dû affronter, en ressort aussi, " en filigrane, la capacité du système scolaire de leur époque de répondre à cette demande» (p. 82). Qu'en a-t-il été pour les trois cadettes?

Les parcours scolaires et professionnels de Dalila, Amel et Nadia (chap. 3) ressemblent certes à ceux de leurs aînées, puisque toutes trois obtiennent un baccalauréat général ou technologique et poursuivent ensuite des études supérieures. La transmission par les aînées de leurs valeurs sociales et culturelles et le suivi très étroit qu'elles ont exercé sur les scolarités de leurs cadettes, Leïla en particulier, ont ainsi favorisé une certaine continuité.

Des écarts n'en apparaissent pas moins, en partie liés aux évolutions du contexte scolaire. Amel, pour prendre son exemple, s'avère avoir été une enfant turbulente, plus rebelle que ses sœurs aînées; si elle trouve que celles-ci avaient " mis la barre très haut " (p. 51), elle semble aussi, par ses comportements au collège et surtout au lycée, s'être peu à peu " conformée à l'image que se font alors a priori d'elle (et de son groupe d'origine) ceux quelle appelle les "autres", c'est-à-dire ceux qui possèdent le pouvoir de la discréditer (notamment les enseignants) "(p. 103). La fronde qu'elle mène en classe de première, qu'elle cache prudemment à sa sœur Leïla, manque d'entraîner son renvoi du lycée... Elle obtiendra toutefois son baccalauréat, fera, comme toutes ses sœurs, des études supérieures et, grâce à son investissement dans les activités d'animation, trouvera sa voie professionnelle comme assistante sociale.

Les cinq filles de la fratrie ont ainsi connu des mobilités sociales ascendantes puisque toutes ont accédé à des emplois intermédiaires, voire supérieurs. Cette mobilité sociale se double d'une mobilité géographique (chap. 6) : alors qu'en 2012, elles habitaient toutes loin du domicile de leurs parents, dont quatre en région parisienne, deux des trois garçons résidaient dans le voisinage de leurs parents.

Il est vrai que les scolarités des trois garçons n'ont pas suivi le modèle de celles de leurs sœurs aînées : "l'école n'a pas été leur priorité dans leur enfance et leur adolescence " (p. 143) Si les moindres attentes scolaires des parents à leur égard, une certaine mansuétude de leur mère, notamment à l'égard de l'aîné, la plus grande liberté dont ils jouissaient ou les pressions constantes de leurs pairs - et, parmi eux, des décrocheurs - ont certainement joué un rôle important dans la pente de leurs scolarités, ils n’en ont pas moins réussi à s'insérer de manière plutôt satisfaisante dans la vie professionnelle (chap. 5, 6 et 7). Les 
trois travaillent et malgré certaines vicissitudes - le parcours délinquant de Rachid ou ses déboires lorsqu'il tente avec son frère Mounir de se mettre à son compte - occupent des positions relativement stabilisées.

Comment rendre compte de ce paradoxe ? Il apparaît notamment que, très tôt, les garçons ont cherché à travailler pendant leurs scolarités : du fait de la situation de précarité et du relatif dénuement dans lesquels la famille avait survécu après la mise en invalidité du père et avant que la mère ne trouve un emploi, les garçons avaient cherché à travailler « à côté de l'école» (p. 145).

C'est ainsi que Rachid, par exemple, est allé travailler sur les marchés dès l'entrée au collège. D'avoir travaillé très tôt les a préparés à affronter le marché du travail, mais leur moindre réussite scolaire explique aussi leur moindre mobilité sociale et, certainement aussi, leur moindre mobilité géographique. Si Rachid a quitté plus d'une fois le domicile parental au cours de sa jeunesse quelque peu chaotique, il est toujours revenu, dit-il, «chez maman-papa" (p. 28). Et comme son frère Mounir, il habite désormais à proximité de ses parents.

Seul Azzedine, puissamment aidé par sa sœur Leïla, s'est éloigné durablement du domicile parental pour s'installer en région parisienne. C'est Leïla, en effet, qui lui a permis de sortir de la galère et d'accéder à un emploi de conducteur de bus à la RATP. Les sœurs aînées ont ainsi pu faire profiter leurs frères de leur expérience et des ressources qu'elles ont accumulées pour faciliter une insertion professionnelle des garçons plus conforme aux espérances de leur père - ne pas travailler avec la pioche!

\section{Face aux soubresauts de l'actualité}

Les trois garçons ont aussi bénéficié de l'attention de leur père qui, aux dires de Samira, "leur parlait beaucoup» (p. 207). Ils n'en ont pas moins dû affronter des difficultés auxquelles leurs sœurs ont échappé le plus souvent, notamment les diverses formes de discrimination dont les garçons font plus souvent l'objet que les filles. Azzedine fait ainsi état d'un contrôle d'identité, dont il a fait l'objet à Paris en 2013, alors qu'il travaille déjà à la RATP : "Mais Monsieur... Fallait me dire que vous étiez de la RATP... " (p. 204) lui dit finalement l'agent... Si les trois frères n'ont jamais voulu se poser en victime de tels agissements, ils n'en sont pas moins confrontés, comme leurs sœurs cette fois, au climat qui s'installe en France après les attentats de 2015 (chap. 8).

Les sœurs aînées avaient contribué à ancrer la famille à gauche alors que les garçons se tenaient "plus ou moins à distance du fait politique " (p. 234) et si Nadia, la plus jeune des cadettes, déçue comme ses frères et sœurs par le quinquennat Hollande, finit par passer à droite (chap. 9), la plupart des membres de la fratrie ont voté à gauche au premier tout de l'élection présidentielle de 2017. 
Au-delà de leurs comportements électoraux, se pose plus fondamentalement la question de leurs réactions aux formes de radicalisation des comportements des jeunes issus de familles maghrébines. Dalila et Nadia, revenues s'installer à proximité de leurs parents, constatent avec effarement les transformations de leur quartier. Nadia : "La moitié (des garçons) ils sont barbus... La moitié des filles, elles sont voilées complètes... Vous discutez avec eux, ils n'ont aucun sujet de conversation, ils se réferent tout le temps à la religion... la religion... la religion... " (p. 263). L'enquête est ainsi infléchie par l'actualité des attentats de 2015 (chap. 10). Le débat au sein de la fratrie sur les usages de l'islam apparaît alors fortement marqué par l'appartenance des uns et des autres à des générations différentes (chap. 11).

\section{Retour sur l'enquête}

Si nous avons rendu compte très rapidement de la troisième partie du livre (chap. 8, 9, 10 et 11), ce n'est certes pas parce que nous la considérons moins intéressante que les deux précédentes. Nous souhaitons vivement que le lecteur s'y rapporte afin de saisir dans leur complexité et leurs nuances les positionnements des membres de la fratrie sur les questions politiques et religieuses. Mais, surtout, nous souhaitons insister, dans cette note, sur ce qui nous paraît un point décisif de l'argumentation de Stéphane Beaud sur l'intégration sociale réussie des enfants de la famille Belhoumi : l'intérêt d'une présentation riche et détaillée des parcours tant scolaires que professionnels des enfants Belhoumi. L'intérêt majeur du livre nous paraît en effet résider dans le fait qu'il nous offre la possibilité d'appréhender plus finement ces parcours et de mieux comprendre les processus à l'œuvre. L'histoire de la fratrie Belhoumi est celle d'enfants issus d'un milieu populaire qui se hissent plus ou moins facilement et plus ou moins nettement à des positions sociales plus confortables que celle de leurs parents ${ }^{2}$. Si les garçons ne gravissent qu'une toute petite marche en accédant à des emplois d'exécution stabilisés, alors que leur père avait travaillé sur un chantier comme manœuvre, les filles gravissent plusieurs marches en accédant, du fait de leur réussite scolaire, à des postions intermédiaires, voire supérieures. Ce faisant, les enfants de la famille Belhoumi concrétisent le vœu de leur père : travailler avec le stylo plutôt qu'avec la pioche! Et nous sommes tentés d'ajouter : concrétisent le projet de toute aventure migratoire, à savoir accéder à de meilleures conditions d'existence. Là est l'histoire ordinaire d'une famille algérienne ordinaire venue s'installer en France.

\footnotetext{
2 Stéphane Beaud rappelle ce résultat de l'enquête TeO (Trajectoires et Origines) : "La mobilité intergénérationnelle ascendante dans les familles maghrébines en France sur la période 1970-2010 est un résultat révélé par les (rares) enquêtes quantitatives disponibles. Selon l'enquête Te $O$ de 2016, $25 \%$ des enfants d'immigrés maghrébins qui se trouvaient en emploi en 2008 (au moment de la passation du questionnaire) sont devenus cadres supérieurs (8\%) ou cadres moyens/professions intermédiaires (18\%). Ce processus décisif a pour seul défaut - si l'on peut dire - d'être peu spectaculaire et moins sujet des Unes des journaux qui réclament la "fait-diversification" de la société et la dramatisation de l'information. " (p. 321)
} 
Nous voici toutefois renvoyés à la question de méthode : l'étude fouillée d'un cas particulier permet-elle de généraliser les conclusions qu'il est possible de tirer de l'analyse fine des données recueillies au cours de l'enquête ? Dans la conclusion de son livre, Stéphane Beaud affirme que "le cas de la famille Belhoumi est bien "exemplaire" en ce qu'il condense un ensemble de processus qui "travaillent" le groupe des enfants d'immigrés algériens (et, par extension, maghrébins." (p. 317).

Les parcours similaires ou différents des enfants de la fratrie témoignent effectivement de la prégnance de la réussite scolaire ou de la différence de comportement des garçons et des filles, mais posent aussi « la question des conditions sociales et historiques de possibilité de la mobilité sociale - c'est-à-dire des facteurs facilitant, versus ceux bloquant, le type de parcours "exemplaires" qu'incarnent ici les filles aînées." (p. 319).

Peuvent être évoqués des facteurs moins souvent signalés tels que les différences de comportement à l'égard de leurs élèves de générations successives d'enseignants, les solidarités familiales ou encore le racisme, diversement vécu par les garçons et les filles. Plus souvent mentionné : l'effet des conjonctures économiques sur les conditions objectives d'intégration des populations migrantes.

Apparaît ainsi clairement la nécessité de saisir les migrations encastrées dans les contextes particuliers qui en déterminent tant la possibilité que les formes de l'intégration des migrants dans les sociétés d'accueil. Mais il apparaît tout aussi clairement que les enfants de migrants constituent désormais un groupe social clivé du fait, précisément, des mobilités différentes auxquelles ils peuvent accéder. "...une fraction importante du groupe des "jeunes d'origine maghrébine» appartient désormais aux classes moyennes et populaires. Elle doit coexister dans la société française avec le groupe des jeunes de même origine socialement exclus ou "désaffliés", qui risque toujours d'entraver la quête de respectabilité de ses membres, pesant ainsi en permanence sur son destin social. » (p. 322), voire de l'occulter...

\section{Référence de l'ouvrage :}

Stéphane Beaud (2018), La France des Belhoumi. Portraits de famille (1977-2017), Paris, Éditions La Découverte. 